\title{
ON THE DETERMINANT OF ELLIPTIC BOUNDARY VALUE PROBLEMS ON A LINE SEGMENT
}

\author{
D. BURGHELEA, L. FRIEDLANDER, AND T. KAPPELER
}

(Communicated by Jeffrey B. Rauch)

\begin{abstract}
In this paper we present a formula for the determinant of a matrixvalued elliptic differential operator of even order on a line segment $[0, T]$ with boundary conditions.
\end{abstract}

\section{INTRODUCTION AND SUMMARY OF THE RESULTS}

In this paper we present a formula for the determinant of a matrix-valued elliptic differential operator of even order on a line segment $[0, T]$ with boundary conditions. In order to state our results we introduce the following notation:

(1) Denote by $\mathscr{A}=\sum_{k=0}^{2 n} a_{k}(x) D^{k}$ a differential operator, $D=D_{x}=-i \frac{d}{d x}$, where the coefficients are complex-valued $r \times r$ matrices depending smoothly on $x, 0 \leq x \leq T$. The leading coefficient $a_{2 n}(x)$ is assumed to be nonsingular and to have $\theta$ as a principal angle, i.e. $R_{\theta} \cap \operatorname{Spec} a_{2 n}(x)=\phi$ for $0 \leq x \leq T$, where $R_{\theta}:=\left\{\rho e^{i \theta} \in \mathbb{C} \mid 0 \leq \rho<\infty\right\}$.

(2) We impose boundary conditions of the form

$$
\ell_{j} u(T)=0 d, \quad m_{j} u(0)=0 \quad(1 \leq j \leq n)
$$

where $u \in C^{\infty}\left([0, T] ; \mathbb{C}^{r}\right)$ and $\ell_{j}, m_{j}$ are differential operators of the form

$$
\ell_{j}:=\sum_{k=0}^{\alpha_{j}} b_{j k} d_{x}^{k}, \quad m_{j}:=\sum_{k=0}^{\beta_{j}} c_{j k} d_{x}^{k} \quad\left(d_{x}=\frac{d}{d x}\right)
$$

such that $b_{j k}, c_{j k}$ are constant $r \times r$ matrices with $b_{j \alpha_{j}}=c_{j \beta_{j}}=$ Id and such that the integers $\alpha_{j}, \beta_{j}$ satisfy

$$
0 \leq \alpha_{1}<\alpha_{2}<\cdots<\alpha_{n} \leq 2 n-1, \quad 0 \leq \beta_{1}<\beta_{2}<\cdots<\beta_{n} \leq 2 n-1 .
$$

Example 1. Dirichlet boundary conditions: $\alpha_{D}=\beta_{D}=(0,1, \ldots, n-1)$

$$
b_{D, j k}=c_{D, j k}:= \begin{cases}\text { Id } & \text { if } 1 \leq j \leq n, k=j-1, \\ 0 & \text { otherwise. }\end{cases}
$$

Received by the editors March 4, 1994.

1991 Mathematics Subject Classification. Primary 34L05; Secondary 35S05. 
Example 2. Neumann boundary conditions: $\alpha_{N}=\beta_{N}=(n, n+1, \ldots, 2 n-1)$

$$
b_{N, j k}=c_{N, j k}:= \begin{cases}\text { Id } & \text { if } 1 \leq j \leq n, k=n+j-1, \\ 0 & \text { otherwise. }\end{cases}
$$

For convenience we write $\alpha=\left(\alpha_{1}, \ldots, \alpha_{n}\right),|\alpha|=\sum_{j=1}^{n} \alpha_{j}$ and similarly $\beta$ and $|\beta|$. Boundary conditions of the above form are usually called separated. Let $B=\left(B_{j k}\right)$ and $C=\left(C_{j k}\right), 1 \leq j \leq 2 n, 0 \leq k \leq 2 n-1$, be $2 n \times 2 n$ matrices whose entries are the following $r \times r$ matrices

$$
\begin{aligned}
B_{j k} & := \begin{cases}b_{j k} & \text { if } 1 \leq j \leq n \text { and } 0 \leq k \leq \alpha_{j}, \\
0 & \text { otherwise }\end{cases} \\
C_{j k} & := \begin{cases}c_{j-n, k} & \text { if } n+1 \leq j \leq 2 n \text { and } 0 \leq k \leq \beta_{j-n}, \\
0 & \text { otherwise. }\end{cases}
\end{aligned}
$$

We denote by $A=A_{B, C}$ the operator $\mathscr{A}$ restricted to the space of smooth functions $u:[0, T] \rightarrow \mathbb{C}^{r}$ satisfying the boundary conditions (1.1).

(3) $\zeta$-regularized determinant $\operatorname{Det}_{\theta} A$. In the case where $A$ is not 1-1, define $\operatorname{Det}_{\theta} A=0$. In the case $A$ is $1-1$, one proceeds as follows. As the coefficient $a_{2 n}(x)$ has $\theta$ as a principal angle, there exists $\varepsilon>0$ so that $L_{(\theta-\varepsilon, \theta+\varepsilon)} \cap \operatorname{Spec} a_{2 n}(x)=\emptyset, 0 \leq x \leq T$, where $L_{(\alpha, \beta)}:=\{z \in \mathbb{C} \mid \alpha \leq \arg z \leq$ $\beta\}$. Then the spectrum of $A, \operatorname{Spec} A$, is discrete, $\operatorname{Spec} A=\left\{\lambda_{j}, j \in \mathbb{N}\right\}$, $\left|\lambda_{j}\right| \rightarrow \infty$, and $\operatorname{Spec} A \cap L_{\left(\theta-\varepsilon^{\prime}, \theta+\varepsilon^{\prime}\right)}$ for any $0<\varepsilon<\varepsilon^{\prime}$ is finite.

If $R_{\theta} \cap \operatorname{Spec} A=\phi$, we define $\zeta_{A, \theta}(s)=\sum_{j \geq 1} \lambda_{j}^{-s}=\operatorname{Tr} A^{-s}$ where $s \in \mathbb{C}$, $\operatorname{Re} s>1 / 2 n$ and where the complex powers are defined with respect to the angle $\theta$. It is a well-known fact that $\zeta_{A, \theta}(s)$ admits a meromorphic extension to $\mathbb{C}$ with $s=0$ being a regular point. According to Ray and Singer [RS] one defines $\log \operatorname{Det}_{\theta} A:=-\left.\frac{d}{d s}\right|_{s=0} \zeta_{A, \theta}(s)$. If $R_{\theta} \cap \operatorname{Spec} A \neq \emptyset$, then choose $\theta^{\prime} \in(\theta-\varepsilon, \theta+\varepsilon)$ so that $R_{\theta^{\prime}} \cap \operatorname{Spec} A=\emptyset$, and define $\operatorname{Det}_{\theta} A:=\operatorname{Det}_{\theta^{\prime}}(A)$. It can be easily checked (cf. [BFK1]) that the definition is independent of the choise of $\theta^{\prime}$ in $=(\theta-\varepsilon, \theta+\varepsilon)$.

(4) The fundamental matrix $Y(x)=Y(x, \mathscr{A})$. Denote by $Y(x)=\left(y_{k \ell}(x)\right)$ $(x \in \mathbb{R})$ the fundamental matrix for $\mathscr{A}$. Note that $Y(x)$ is a $2 n \times 2 n$ matrix whose entries $y_{k \ell}(x)(0 \leq k, \ell \leq 2 n-1)$ are $r \times r$ matrices defined by

$$
y_{k \ell}(x):=d_{x}^{k} y_{\ell}(x)
$$

where $y_{\ell}(x)$ denotes the solution of the Cauchy problem $\mathscr{A} y_{\ell}(x)=0, y_{k \ell}(0)=$ $\delta_{k \ell}$ Id . Of particular interest is the $2 n \times 2 n$ matrix $Y(T)$, the evaluation of the fundamental matrix at $x=T$.

(5) Introduce the quantities

$$
g_{\alpha}:=\frac{1}{2}\left(\frac{|\alpha|}{n}-n+\frac{1}{2}\right), \quad h_{\alpha}=\operatorname{det}\left(\begin{array}{ccc}
w_{1}^{\alpha_{1}} & \cdots & w_{n}^{\alpha_{1}} \\
\vdots & & \vdots \\
w_{1}^{\alpha_{n}} & \cdots & w_{n}^{\alpha_{n}}
\end{array}\right)
$$

where $w_{1}, \ldots, w_{n}$ denote the $2 n$th roots of $(-1)^{n+1}$ with $\operatorname{Re} w>0$ given by $w_{k}=\exp \left\{\frac{2 k-n-1}{2 n} \pi i\right\}$. For a $r \times r$ matrix $a$ with principal angle $\theta$ and eigenvalues $\lambda_{1}, \ldots, \lambda_{r}$, denote $(\operatorname{det} a)_{\theta}^{g_{\alpha}}=\prod_{j=1}^{r}\left|\lambda_{j}\right|^{g_{\alpha}} \exp \left\{i g_{\alpha} \arg \lambda_{j}\right\}$ where $\theta-2 \pi<\arg \lambda_{j}<\theta$. 
Example 1. Dirichlet boundary conditions:

$$
g_{\alpha_{D}}=-n / 4, h_{\alpha_{D}}=h_{n}:=\prod_{i>j}\left(w_{i}-w_{j}\right) .
$$

Example 2. Neumann boundary conditions:

$$
g_{\alpha_{N}}=n / 4, h_{\alpha_{N}}=(-1)^{n} h_{n} .
$$

The main result of this paper is

Theorem.

$$
\operatorname{Det}_{\theta} A=K_{\theta} \exp \left\{\frac{i}{2} \int_{0}^{T} \operatorname{tr}\left(a_{2 n}^{-1}(x) a_{2 n-1}(x)\right) d x\right\} \operatorname{det}(B Y(T)-C)
$$

where $K_{\theta} \equiv K_{\theta}(\alpha, \beta)$ is given by

$$
K_{\theta}=\left((-1)^{|\beta|}(2 n)^{n} h_{\alpha}^{-1} h_{\beta}^{-1}\right)^{r}\left(\operatorname{det} a_{2 n}(0)\right)_{\theta}^{g_{\beta}}\left(\operatorname{det} a_{2 n}(T)\right)_{\theta}^{g_{\alpha}} .
$$

Example 1. Dirichlet boundary conditions: $\quad\left|\alpha_{D}\right|=\frac{n(n-1)}{2}$,

$$
K_{\theta}=\left((-1)^{\left|\alpha_{D}\right|}(2 n)^{n} h_{n}^{-2}\right)^{r}\left(\operatorname{det} a_{2 n}(0)\right)_{\theta}^{-\frac{n}{4}}\left(\operatorname{det} a_{2 n}(T)\right)_{\theta}^{-\frac{n}{4}} .
$$

Example 2. Neumann boundary conditions: $\quad\left|\alpha_{N}\right|=\frac{n(n-1)}{2}$,

$$
K_{\theta}=\left((-1)^{\left|\alpha_{D}\right|}(2 n)^{n} h_{\alpha_{N}}^{-2}\right)^{r}\left(\operatorname{det} a_{2 n}(0)\right)_{\theta}^{\frac{n}{4}}\left(\operatorname{det} a_{2 n}(T)\right)_{\theta}^{\frac{n}{4}} .
$$

Corollary. Det $_{\theta} A$ is a complex number independent of $\theta$ up to multiplication with a $2 n$th root of unity.

Remark 1 . In the formula above all terms except the matrix $Y(T)$ are easily computable from the coefficients of $\mathscr{A}, \ell_{i}$ and $m_{j}$. The matrix $Y(T)$ requires the knowledge of the fundamental solutions. The matrix $Y(T)$ and therefore $\operatorname{det}(B Y(T)-C)$ can be calculated numerically within arbitrary accuracy by solving a finite difference equation approximating $\mathscr{A}$. So the determinant $\operatorname{Det}_{\theta} A$ can be calculated numerically within arbitrary accuracy.

Remark 2. Theorem is a companion of the corresponding result on the circle instead of the interval $[0, T]$ which was treated in an earlier paper [BFK1]. Again, the proof of Theorem relies on a deformation argument and explicit computations for certain special operators and special boundary conditions.

Remark 3. Introduce a spectral parameter $\lambda$, and denote the fundamental matrix of $\mathscr{A}+\lambda$ by $Y(x, \lambda)=Y(x, \mathscr{A}+\lambda)$. One then verifies $\operatorname{det}(B Y(T ; \lambda)-C)$ $=0$ iff $\operatorname{Det}_{\theta}(A+\lambda)=0$, i.e. iff $-\lambda$ is an eigenvalue of $A=A_{B}, C$.

Remark 4. First results of the type described in Theorem are due to Dreyfus and Dym [DD] and to Forman [Fol] (cf. also [Fo2]). Forman proved by different methods that the quotient $\operatorname{Det}_{\theta} A / \operatorname{Det}(B Y(T)-C)$ only depends on the principal and subprincipal symbols of $\mathscr{A}$, and the principal symbol of the boundary operators $\ell_{j}, m_{j}(1 \leq j \leq n)$. Our Theorem provides a formula for this quotient. 
Remark 5. Analogous to results obtained in [BFK2], Theorem can be extended to the case where $\mathscr{A}$ is a pseudodifferential operator. The determinant $\operatorname{Det}_{\theta} A$ can be written as a product of local invariants with a Fredholm determinant of a pseudodifferential operator of determinant class, canonically associated to $A$. The Fredholm determinant corresponds to $\operatorname{det}(B Y(T)-C)$ in the case when $\mathscr{A}$ is a differential operator.

\section{AuXILIARY RESULTS}

In this section we collect some auxiliary results needed for the proof of Theorem. First we introduce some additional notation. Denote by $E D O_{2 n} \equiv$ $E D O_{2 n, r}$ the set of all elliptic differential operators $\mathscr{A}$ of order $2 n$ on $[0, T]$ as introduced in Section 1. We identify $E D O_{2 n}$ with the open set $\left\{\left(a_{2 n}, \ldots, a_{0}\right)\right.$ $\left.\in C^{\infty}\left([0, T] \text {, End } \mathbb{C}^{r}\right)^{2 n+1}: \operatorname{det}\left(a_{2 n}(x)\right) \neq 0,0 \leq x \leq T\right\}$ of the Frechet space $C^{\infty}\left([0, T] \text {, End } \mathbb{C}^{r}\right)^{2 n+1}$. Further define $E D O_{2 n, \theta}:=\left\{\mathscr{A} \in E D O_{2 n}: \theta\right.$ is principal angle for $\left.a_{2 n}\right\}$. Clearly $E D O_{2 n ; \theta}$ is an open connected subset in $E D O_{2 n}$. Given $\alpha=\left(\alpha_{1}, \ldots, \alpha_{n}\right) \in \mathbb{Z}^{n}$ with $0 \leq \alpha_{1}<\alpha_{2}<\cdots<\alpha_{n} \leq 2 n-1$, we introduce the space $B D O_{\alpha}$ of operators used to define the boundary conditions:

$$
\begin{array}{r}
B D O_{\alpha}:=\left\{B=\left(b_{j k}\right)_{0 \leq j, k \leq 2 n-1}: \quad b_{j k} \in \operatorname{End} \mathbb{C}^{r}, b_{j \alpha_{j}}=\text { Id },\right. \\
\left.b_{j k}=0 \text { if } k \geq \alpha_{j}+1\right\} .
\end{array}
$$

Given $\alpha, \beta$, we introduce the space

$$
E D O_{2 n ; \alpha ; \beta}:=\left\{A_{B, C}: \mathscr{A} \in E D O_{2 n}, B \in B D O_{\alpha}, C \in B D O_{\beta}\right\}
$$

where $A_{B}, C$ is the restriction of $\mathscr{A}$ to the subspace of functions $u \in$ $C^{\infty}\left([0, T] ; \mathbb{C}^{r}\right)$ satisfying the boundary conditions defined by $B$ and $C$. Similarly introduce $E D O_{2 n, \theta ; \alpha, \beta}=\left\{A_{B, C} \in E D O_{2 n ; \alpha, \beta}: \mathscr{A} \in E D O_{2 n ; \theta}\right\}$. Observe that $\left\{A_{B, C} \in E D O_{2 n ; \theta ; \alpha ; \beta}: A_{B, C}\right.$ is $\left.1-1\right\}$ is open.

Further, denote by $\widehat{E D O_{2 n ; \alpha, \beta}}$ the open subset of $E D O_{2 n ; \alpha, \beta} \times S^{1}$ consisting of pairs $\left(A_{B, C}, \theta\right)$ with $A_{B, C} \in E D O_{2 n ; \theta ; \alpha ; \beta}$. As in [BFK1] we have the following

Proposition 2.1. (1) $\operatorname{Det}_{\theta}\left(A_{B, C}\right)$ is a smooth function on $\widehat{E D O_{2 n ; \alpha ; \beta}}$ and is locally constant in $\theta$.

(2) $\operatorname{Det}_{\theta}\left(A_{B}, C\right)$ is holomorphic when considered as a function on the open subset of injective operators in $E D O_{2 n ; \theta ; \alpha ; \beta}$.

(3) $\operatorname{det}(B Y(T, \mathscr{A})-C)$ is holomorphic on $E D O_{2 n} \times B D O_{\alpha} \times B D O_{\beta}$.

Observe that a necessary and sufficient condition for $A_{B}, C$ to have zero as an eigenvalue is that $\operatorname{det}(B Y(T)-C)=0$, which in view of Proposition 2.1 (3) implies that the subsets of $E D O_{2 n ; \theta ; \alpha ; \beta}$ and $E D O_{2 n, \alpha, \beta}$ consisting of injective operators are open (as we already noticed) and connected, and therefore, $\widehat{E D O}_{2 n ; \alpha ; \beta}$ is open and connected as well.

Let $s:[0, T] \rightarrow G L\left(\mathbb{C}^{r}\right)$ be a smooth map. Given $\mathscr{A} \in E D O_{2 n}$ and boundary operators $\ell_{j}, m_{j}(1 \leq j \leq n)$ introduce $\mathscr{A}_{1}:=s(x)^{-1} \mathscr{A} s(x)$, $\ell_{1 j}:=\left.s(T)^{-1} \ell_{j} s(x)\right|_{x=T}$, and $m_{1 j}:=\left.s(0)^{-1} m_{j} s(x)\right|_{x=0}$. Denote by $\left(B_{1 j k}\right)$ and $\left(C_{1 j k}\right)$ the matrices introduced in Section 1 corresponding to the boundary operators $\left(\ell_{1 j}, m_{1 j}\right)_{1 \leq j \leq n}$ and write $Y_{1}(x)=Y\left(x, \mathscr{A}_{1}\right)$ for short.

Proposition 2.2. $\operatorname{det}\left(B_{1} Y_{1}(T)-C_{1}\right)=\left(\operatorname{det} s(0) s(T)^{-1}\right)^{n} \operatorname{det}(B Y(T)-C)$. 
Proof. Let $L=L(x)$ be a $2 n \times 2 n$ matrix with entries $L_{k \ell}$ which are the following $r \times r$ matrices $(0 \leq k, \ell \leq 2 n-1)$

$$
L_{k \ell}:=\left(\begin{array}{l}
k \\
\ell
\end{array}\right) d_{x}^{k-\ell} s(x) \text { if } k \geq \ell ; \quad L_{k \ell}=0 \quad \text { if } k<\ell .
$$

Thus we obtain

$$
B_{1}=\operatorname{diag}\left(s(T)^{-1}, \ldots, s(T)^{-1}\right) B L(T)
$$

where $\operatorname{diag}\left(s(T)^{-1}, \ldots, s(T)^{-1}\right)$ is a $2 n \times 2 n$ diagonal matrix whose entries on the diagonal are all equal to the $r \times r$ matrix $s(T)^{-1}$. Similarly, one obtains

$$
C_{1}=\operatorname{diag}\left(s(0)^{-1}, \ldots, s(0)^{-1}\right) C L(0) .
$$

Further, by a straightforward computation, $Y_{1}$ is given by

$$
Y_{1}(x)=L(x)^{-1} Y(x) L(0) \text {. }
$$

Thus

$$
\begin{aligned}
B_{1} Y_{1}(T)-C_{1}= & \operatorname{diag}\left(s(T)^{-1}, \ldots, s(T)^{-1}, s(0)^{-1}, \ldots, s(0)^{-1}\right) \\
& \cdot[B Y(T)-C] L(0)
\end{aligned}
$$

Now observe that $\operatorname{det} L(0)=(\operatorname{det} s(0))^{2 n}$ as $L(0)$ is lower triangular with diagonal entries all equal to the $r \times r$ matrix $s(0)$. This implies that

$$
\operatorname{det}\left(B_{1} Y_{1}(T)-C_{1}\right)=\left(\operatorname{det} s(0) s(T)^{-1}\right)^{n} \cdot \operatorname{det}(B Y(T)-C) .
$$

Next consider for $A=A_{B, C}$ in $E D O_{2 n ; \theta ; \alpha ; \beta}$ and $\Phi \in C^{\infty}\left([0, T], G L_{r}(\mathbb{C})\right)$ the generalized $\zeta$-function $\zeta_{\Phi, A ; \theta}(s):=\operatorname{tr} \Phi A_{\theta}^{-s}$. Again this is a function which is holomorphic in $\operatorname{Re} s>\frac{1}{2 n}$ and has a meromorphic extension to the whole complex plane. Moreover $s=0$ is a regular point. Recall that we have introduced $g_{\alpha}:=\frac{1}{2}\left(\frac{|\alpha|}{n}-n+\frac{1}{2}\right)$, and similarly $g_{\beta}$.

Proposition 2.3.

$$
\zeta_{\Phi, A ; \theta}(0)=g_{\beta} \operatorname{tr} \Phi(0)+g_{\alpha} \operatorname{tr} \Phi(T)
$$

As an immediate consequence we obtain

Corollary 2.4. $\zeta_{A ; \theta}(0)=r\left(g_{\alpha}+g_{\beta}\right)=r\left(\frac{|\alpha|+|\beta|}{2 n}-n+1\right)$.

Proof (Proposition 2.3). We first prove that there are numbers $\tilde{g}_{\alpha}, \tilde{g}_{\beta} \in \mathbb{C}$ which only depend on $\alpha$ and $\beta$ respectively such that $(2.1)$ holds. The actual values of $\tilde{g}_{\alpha}, \tilde{g}_{\beta}$ are computed at the end of section 3 by considering the case $\Phi(x) \equiv K$ with $K>1, \mathscr{A}=D^{n}+\lambda, \theta=\pi$. In the course of the proof we use a number of results due to Seeley [Se1,2]. For the convenience of the reader we partly keep Seeley's notation. For simplicity, we write $\zeta(s)=\zeta_{\Phi, A ; \theta}(s)$. According to [Se2], the value $\zeta(0)$ consists of a sum of two terms, $\zeta(0)=I+I I$ where $I$ represents the contribution to $\zeta(0)$ of the resolvent of $\mathscr{A}-\lambda$ and $I I$ represents a correction term due to the boundary conditions. According to [BFK1, p. 8],

$$
I=-\frac{e^{i \theta}}{4 \pi n} \sum_{\tau= \pm 1} \int_{0}^{T} d x \int_{0}^{\infty} d r \operatorname{tr}\left\{\Phi(x) c_{-2 n-1}\left(x, \tau, r e^{i \theta}\right)\right\}
$$

where $c_{-2 n-1}(x, \tau, \lambda)$ comes from the expansion of the symbol

$$
r(x, \tau, \lambda)=c_{-2 n}(x, \tau, \lambda)+c_{-2 n-1}(x, \tau, \lambda)+\cdots
$$


of the parametrix for $\mathscr{A}-\lambda=\left(a_{2 n}(x) D^{2 n}-\lambda\right)+\sum_{j=0}^{2 n-1} a_{j}(x) D^{j}$ and is given by

$c_{-2 n-1}(x, \tau, \lambda)=-\tau^{2 n-1} c_{-2 n} a_{2 n-1} c_{-2 n}-i 2 n \tau^{4 n-1} c_{-2 n} a_{2 n} c_{-2 n}\left(\frac{d}{d x} a_{2 n}\right) c_{-2 n}$

where $c_{-2 n} \equiv c_{-2 n}(x, \tau, \lambda)=\left(a_{2 n}(x) \tau^{2 n}-\lambda\right)^{-1}$.

As in [BFK1], Proposition 2.8, in view of the fact that $c_{-2 n-1}$ is odd in $\tau$, we conclude $I=0$. From [Se2], p. 968, it follows that $I I$ is of the form

$$
I I=\operatorname{tr}\left\{\Delta_{0}^{\prime}(0) \Phi(0)+\Delta_{T}^{\prime}(0) \Phi(T)\right\}
$$

where $\Delta_{0}^{\prime}(s)$ and $\Delta_{T}^{\prime}(s)$ are smooth functions described below. Let us first consider the scalar case, $r=1$. In first approximation the kernel $r(x, y, \lambda)$ of $\left(A_{B}, C-\lambda\right)^{-1}$ is given by

$$
\frac{1}{2 \pi} \int_{-\infty}^{+\infty}\left(a_{2 n}(x) \tau^{2 n}-\lambda\right)^{-1} e^{i(x-y) \tau} d \tau+r_{0}(x, y, \lambda)+r_{T}(x, y, \lambda)
$$

where $r_{0}(x, y, \lambda)$ and $r_{T}(x, y, \lambda)$ are correction terms so that in first approximation $r(x, y, \lambda)$ satisfies the boundary conditions at $x=0$ and $x=T$. Let us explain how to obtain $r_{0}(x, y, \lambda)$; for $r_{T}(x, y, \lambda)$ one proceeds in a similar fashion. Consider the boundary value problem

$$
\left(a D^{2 n}-\lambda\right) u=0
$$

with the boundary condition

$$
\lim _{x \rightarrow \infty} u(x)=0 ; \quad D^{\beta_{j}} u(0)=-\left(a \tau^{2 n}-\lambda\right)^{-1} \tau^{\beta_{j}} e^{-i y \tau}
$$

where $a=a_{2 n}(0)$ and $D=\frac{1}{i} \frac{d}{d x}$. The solution $u(x)=u(x, \tau, y, \lambda)$ of the boundary value problem $(2.2)-(2.3)$ is given by $u(x)=\sum_{\nu=1}^{n} u_{\nu} e^{i x(-\lambda / a)^{1 / 2 n} w_{\nu}}$ where $w_{\nu}(1 \leq \nu \leq n)$ are the $2 n$th roots of -1 with strictly positive imaginary part and where $\left(-\frac{\lambda}{a}\right)^{1 / 2 n}=\left(-\frac{\lambda}{a}\right)_{\pi}^{1 / 2 n}=\left(\frac{|\lambda|}{|a|}\right)^{1 / 2 n} e^{i(\theta-\pi-\arg a) / 2 n}$ with $\lambda=|\lambda| e^{i \theta}$ and $\theta-2 \pi<\arg a<\theta$. The coefficients $u_{\nu}=u_{\nu}(\tau, y, \lambda)$ are then determined by (2.3)

$$
\sum_{\nu=1}^{n} u_{\nu}\left(-\frac{\lambda}{a}\right)^{\beta_{j} / 2 n} w_{\nu}^{\beta_{j}}=-\tau^{\beta_{j}}\left(a \tau^{2 n}-\lambda\right)^{-1} e^{-i y \tau}
$$

Thus

$$
u_{\nu}=-\sum_{j=1}^{n} \mathscr{H}_{\nu_{j}}(-\lambda / a)^{-\beta_{j} / 2 n} \tau^{\beta_{j}}\left(a \tau^{2 n}-\lambda\right)^{-1} e^{-i y \tau}
$$

with $\mathscr{H}_{\nu_{j}}$ defined by

$$
\sum_{j=1}^{n} \mathscr{H}_{\nu_{j}} w_{k}^{\beta_{j}}=\delta_{\nu k}
$$

The term $r_{0}(x, y, \lambda)$ is then given by

$$
r_{0}(x, y, \lambda)=\sum_{\nu=1}^{n} e^{i x(-\lambda / a)^{1 / 2 n}} w_{\nu} \sum_{j=1}^{n} \mathscr{H}_{\nu j} \frac{1}{i}(-\lambda / a)^{-\beta_{j} / 2 n} \mathscr{I}
$$


where $\mathscr{I}$ is the sum of residues

$$
\mathscr{I}=\sum_{k=1}^{n} \operatorname{Re} s_{\tau_{k}=(-\lambda / a)^{1 / 2 n} \bar{w}_{k}}\left\{\tau^{\beta_{j}}\left(a \tau^{2 n}-\lambda\right)^{-1} e^{-i y \tau}\right\}
$$

of $\tau^{\beta_{j}}\left(a \tau^{2 n}-\lambda\right)^{-1} e^{-i y \tau}$ in the lower half plane. One obtains

$$
\mathscr{I}=\sum_{k=1}^{n}\left((-\lambda / a)^{1 / 2 n} \bar{w}_{k}\right)^{\beta_{j}-(2 n-1)} \frac{1}{2 n a} \exp \left\{-i y(-\lambda / a)^{1 / 2 n} \bar{w}_{k}\right\}
$$

Summarizing one obtains

$$
r_{0}(x, y, \lambda)=\frac{i}{2 n a}(-\lambda / a)^{-(2 n-1) / 2 n} \sum_{\nu, j, k} \mathscr{H}_{\nu j} \bar{w}_{k}^{\beta+1} \exp \left\{i(-\lambda / a)^{1 / 2 n}\left(x w_{\nu}-y \bar{w}_{k}\right)\right\}
$$

Following Seeley, we now define for $\operatorname{Re} s>0$

$$
\Delta_{0}^{\prime}(s):=\int_{0}^{T / 2} d x \frac{1}{2 \pi i} \int_{\Gamma_{\theta}} d \lambda \lambda^{-s} r_{0}(x, x, \lambda)
$$

where $\Gamma_{0}$ is the contour that goes from $\infty$ to 0 along the lower side of ray $\left\{r e^{i \theta}: r>0\right\}$, goes around the origin and then returns to $\infty$ along the upper side of the ray $\left\{r e^{i \theta}: r>0\right\}$. By a standard computation,

$$
\begin{aligned}
& \frac{1}{2 \pi i} \int_{\Gamma_{\theta}} d \lambda \lambda^{-s} r_{0}(x, x, \lambda) \\
& \quad=a^{-s} e^{-i \pi n s} \frac{\sin \pi s}{\pi} \Gamma(1-2 n s) \sum_{\nu, j, k} \mathscr{H}_{\nu j} \bar{w}_{k}^{\beta_{j}+1}\left(\left(w_{\nu}-\bar{w}_{k}\right) x\right)^{-1+2 n s}
\end{aligned}
$$

and therefore

$$
\Delta_{0}^{\prime}(0)=\frac{1}{2 n} \sum_{\nu, j, k} \mathscr{H}_{\nu j} \frac{\bar{w}_{k}^{\beta_{j}+1}}{w_{\nu}-\bar{w}_{k}}
$$

In the case $r \geq 2$, we first treat the case where all eigenvalues of $a_{2 n}(0)$ are different which can be easily reduced to scalar case $r=1$. By a continuity argument we then conclude that

$$
\tilde{g}_{\beta}=\frac{1}{2 n} \sum_{\nu, j, k} \mathscr{H}_{\nu j}(\beta) \bar{w}_{k}^{\beta_{j}+1}\left(w_{\nu}-\bar{w}_{k}\right)^{-1}
$$

where $\mathscr{H}_{\nu j}=\mathscr{H}_{\nu j}(\beta)$ are determined by (2.4). Similarly one obtains

$$
\tilde{g}_{\alpha}=\frac{1}{2 n} \sum_{\nu j, k} \mathscr{H}_{\nu_{j}}(\alpha) \bar{w}_{k}^{\alpha_{j}+1}\left(w_{\nu}-\bar{w}_{k}\right)^{-1} .
$$

\section{Proof of Theorem 1}

For the proof of Theorem we need two deformation results. The first one is the analogue of Proposition 3.1 in [BFK1] and proved in a similar way (cf. also [DD] and [Fol]). 
Proposition 3.1. Suppose $\mathscr{A}=\sum_{k=0}^{2 n} a_{k}(x) D^{k}$ and $\mathscr{A}^{\prime}=\sum_{k=0}^{2 n} a_{k}^{\prime}(x) D^{k}$ are in $E D O_{2 n ; \theta}$ with $a_{2 n}=a_{2 n}^{\prime}$ and $a_{2 n-1}=a_{2 n-1}^{\prime}$. Then, for $B \in B D O_{\alpha}$ and $C \in B D O_{\beta}$

$$
\operatorname{Det}_{\theta}\left(A_{B, C}\right) \operatorname{det}\left(B Y\left(T, \mathscr{A}^{\prime}\right)-C\right)=\operatorname{Det}_{\theta}\left(A_{B, C}^{\prime}\right) \operatorname{det}(B Y(T, \mathscr{A})-C) .
$$

The second result concerns a deformation of the boundary conditions. Consider boundary operators $\left(1 \leq j \leq n, d_{x}=\frac{d}{d x}\right)$

$$
\ell_{j}=\sum_{k=0}^{\alpha_{j}} b_{j k} d_{x}^{k}, \quad m_{j}=\sum_{k=0}^{\beta_{j}} c_{j k} d_{x}^{k} ; \quad b_{j \alpha_{j}}=c_{j \beta_{j}}=\mathrm{Id}
$$

and

$$
\ell_{j}^{\prime}:=d_{x}^{\alpha_{j}}, \quad m_{j}^{\prime}=d_{x}^{\beta_{j}} .
$$

Form the matrices $B, C$ and $B^{\prime}, C^{\prime}$ as in Section 1.

Proposition 3.2. Fix $\mathscr{A} \in E D O_{2 n ; \theta}$. Then

$$
\operatorname{Det}_{\theta}\left(A_{B^{\prime}, C^{\prime}}\right) \operatorname{det}(B Y(T)-C)=\operatorname{Det}_{\theta}\left(A_{B, C}\right) \operatorname{det}\left(B^{\prime} Y(T)-C^{\prime}\right) \text {. }
$$

Proof. Without loss of generality we may assume that both $A_{B, C}$ and $A_{B^{\prime}, C^{\prime}}$ are injective. Note that $\left\{A_{\widetilde{B}, \widetilde{C}}: A_{\widetilde{B}, \widetilde{C}}\right.$ is $\left.1-1, \widetilde{B} \in B D O_{\alpha}, \widetilde{C} \in B D O_{\beta}\right\}$ is arcwise connected in $B D O_{\alpha} \times B D O_{\beta}$. Define, for $0 \leq t \leq 1$,

$$
\ell_{t j}=d_{x}^{\alpha_{j}}+t \sum_{k=0}^{\alpha_{j}-1} b_{j k} d_{x}^{k}, \quad c_{t j}=d_{x}^{\beta_{j}}+t \sum_{k=0}^{\beta_{j}-1} c_{j k} d_{x}^{k}
$$

such that, with $B_{t}$ and $C_{t}$ the corresponding matrices in $B D O_{\alpha}$ and $B D O_{\beta}$,

$$
\begin{gathered}
A_{B_{t}, C_{t}} \text { is } 1-1 \text { for } 0 \leq t \leq 1 ; \\
\left(B_{0}, C_{0}\right)=\left(B^{\prime}, C^{\prime}\right), \quad\left(B_{1}, C_{1}\right)=(B, C) .
\end{gathered}
$$

Introduce

$$
w(t):=\frac{\frac{d}{d t} \operatorname{Det}_{\theta}\left(A_{B_{t}}, C_{t}\right)}{\operatorname{Det}_{\theta}\left(A_{B_{t}}, C_{t}\right)}, \quad \delta(t):=\frac{\frac{d}{d t} \operatorname{det}\left(B_{t} Y(T)-C_{t}\right)}{\operatorname{det}\left(B_{t} Y(T)-C_{t}\right)} .
$$

The claimed result follows once we show that $w(t)=\delta(t) \quad(0 \leq t \leq 1)$. Let us first consider $\delta(t)$. Denote by $P_{t}$ the Poisson operator corresponding to the boundary value problem defined by $\left(B_{t}, C_{t}\right)$. Then $P_{t}$ is given by $P_{t}=$ $Y(x)\left(B_{t} Y(T)-C_{t}\right)^{-1}$ and

$$
\begin{aligned}
\delta(t) & =\operatorname{tr}\left\{\left(\dot{B}_{t} Y(T)-\dot{C}_{t}\right)\left(B_{t} Y(T)-C_{t}\right)^{-1}\right\} \\
& =\operatorname{tr}\left(\left(\dot{\ell}_{t j}, \dot{m}_{t j}\right)_{1 \leq j \leq n} P_{t}\right)
\end{aligned}
$$

when $=\frac{d}{d t}$ and $\left(\dot{\ell}_{t j}, \dot{m}_{t j}\right)_{1 \leq j \leq n}$ is the operator associating to a section $u$ the boundary values $\left(\dot{\ell}_{t j} u(T), \dot{m}_{t j} u(0)\right)_{1 \leq j \leq n}$.

Next we consider $w(t)$; with the notation $A_{t}=A_{B_{t}}, C_{t}$,

$$
w(t)=F \cdot p_{\cdot s=0} \operatorname{tr}\left(A_{t}^{*} A_{t}^{-1-s}\right)
$$

where $F . p . s=0$ denotes the finite part at $s=0$. In order to evaluate $A_{t}^{-1} A_{t}^{*}=$ $-\left(A_{t}^{-1}\right)^{\cdot} A_{t}$, consider for a fixed section $u:[0, T] \rightarrow \mathbb{C}^{r}$ the section $v_{t}:=A_{t}^{-1} u$, i.e. $v_{t}$ satisfies

$$
\mathscr{A} v_{t}=u, \quad B_{t} v_{t}(T)=0, \quad C_{t} v_{t}(0)=0 .
$$


Taking derivatives with respect to $t$ we obtain

$$
\mathscr{A} v_{t}^{\cdot}=0, \quad \ell_{t j} v_{t}^{\cdot}(T)=-\ell_{t j}^{\cdot} v_{t}(T), \quad m_{t j} v_{t}^{\cdot}(0)=-m_{t j}^{\cdot} v_{t}(0) \quad(1 \leq j \leq n) .
$$

Thus $v_{t}^{\cdot}=-P_{t}\left(\ell_{t j}^{\cdot} v_{t}(T), m_{t j}^{\cdot} v_{t}(0)\right)_{1 \leq j \leq n}$ where $P_{t}$ again denotes the Poisson operator. Thus we have proved that $\left(A_{t}^{-1}\right)^{\cdot}=-P_{t}\left(\ell_{t j}^{\cdot}, m_{t j}^{\cdot}\right)_{1 \leq j \leq n} A_{t}^{-1}$. Note that $\left(A_{t}^{-1}\right)^{\cdot} A_{t}=-P_{t}\left(\ell_{t j}^{\cdot}, m_{t j}^{\cdot}\right)_{1 \leq j \leq n}$ is a singular Green's operator of order $\leq-2$ and then of trace class. Thus

$$
w(t)^{\cdot}=\operatorname{tr} P_{t}\left(\ell_{k j}^{\cdot}, m_{i j}^{\cdot}\right)_{1 \leq j \leq n} .
$$

Proof of Theorem. We have to prove that

$$
\begin{aligned}
f_{\theta}\left(A_{B, C}\right):= & \operatorname{Det}_{\theta}\left(A_{B, C}\right)-K_{\theta} \exp \left\{\frac{i}{2} \int_{0}^{T} \operatorname{tr}\left(a_{2 n}(x)^{-1} a_{2 n-1}(x)\right) d x\right\} \\
& \cdot \operatorname{det}(B Y(T)-C)
\end{aligned}
$$

vanishes identically on $\left\{A_{B, C} \in E D O_{2 n ; \theta ; \alpha ; \beta}: A_{B, C}\right.$ is $\left.1-1\right\}$. First observe that it suffices to consider the case $\theta=\pi$ : For $\mathscr{A}$ in $E D O_{2 n ; \theta}, e^{i(\pi-\theta)} \mathscr{A} \in$ $E D O_{2 n ; \pi}$ we have $\log \operatorname{Det}_{\pi}\left(e^{i(\pi-\theta)} A_{B, C}\right)=\log \operatorname{Det}_{\theta}\left(A_{B}, C\right)+\zeta_{A, \theta}(0) \log e^{i(\pi-\theta)}$ and $\log K_{\theta}\left(e^{i(\pi-\theta)} \mathscr{A}\right)=\log K_{\pi}(t)+r\left(g_{\beta}+g_{\alpha}\right) i(\pi-\theta)$; thus Corollary 2.4 allows to conclude the result as soon as we check it for $\theta=\pi$.

To make writing easier, let $f \equiv f_{\pi}, K \equiv K_{\pi}, \theta \equiv \pi$.

Deformation 1. Consider the factorization $\mathscr{A}=a_{2 n}\left(D^{2 n}+\mathscr{H}\right)$ where $\mathscr{H}$ is a differential operator with ord $\mathscr{H} \leq 2 n-1$. Consider the 1-parameter family $(0 \leq t \leq 1)$

$$
\mathscr{A}_{t}:=\alpha_{t}\left(D^{2 n}+\mathscr{H}\right), \quad A_{t}:=A_{t, B, C}
$$

when $\alpha_{t}(x)=t a_{2 n}(x)+(1-t)$.

Clearly $\theta=\pi$ is a principal angle for $\alpha_{t}$ and $A_{t}$ is $1-1$ for $0 \leq t \leq$ 1. Moreover $A_{t}^{*}=\left(a_{2 n}(x)-1\right)\left(t a_{2 n}(x)+(1-t)\right)^{-1} A_{t}$. Thus, with $\bar{w}(t)=$ $\log \operatorname{Det}_{\pi} A_{t}$ and Proposition 2.3

$$
\begin{aligned}
w(t)^{\circ}= & F \cdot p \cdot s=0 \operatorname{tr}\left(\left(a_{2 n}(x)-1\right)\left(t a_{2 n}(x)+(1-t)\right)^{-1} A(t)^{-s}\right) \\
= & g_{\beta} \operatorname{tr}\left[\left(a_{2 n}(0)-1\right)\left(\operatorname{ta}_{2 n}(0)+(1-t)\right)^{-1}\right] \\
& +g_{\alpha} \operatorname{tr}\left[\left(a_{2 n}(T)-1\right)\left(t a_{2 n}(T)+(1-t)\right)^{-1}\right] \\
= & \frac{d}{d t}\left\{g_{\beta} \log \operatorname{det}\left[\operatorname{ta}_{2 n}(0)+(1-t)\right]\right. \\
& \left.+g_{\alpha} \log \operatorname{det}\left[\operatorname{ta}_{2 n}(T)+(1-t)\right]\right\} .
\end{aligned}
$$

Thus

$\log \operatorname{Det}_{\pi} A_{1}-\log \operatorname{Det}_{\pi} A_{0}=\int_{0}^{1} w(t)^{\cdot} d t=g_{\beta} \log \operatorname{det}\left(a_{2 n}(0)\right)+g_{\alpha} \log \operatorname{det}\left(a_{2 n}(T)\right)$.

Hence we may and will assume that $a_{2 n}(x) \equiv \mathrm{Id}$.

Deformation 2. Define $s \in C^{\infty}\left([0, T]\right.$; End $\left.\mathbb{C}^{r}\right)$ by

$$
\frac{d}{d x} s(x)=\frac{i}{2 n} a_{2 n-1}(x) s(x) \quad(0 \leq x \leq T) ; \quad s(0)=\mathrm{Id} .
$$


Observe that $\operatorname{det}(s(x))=\exp \left\{\frac{i}{2 n} \int_{0}^{x} \operatorname{tr}\left(a_{2 n-1}(y)\right) d y\right\} \neq 0$ for $0 \leq x \leq T$ and therefore $s(x) \in G L_{r}(\mathbb{C})$. Now consider $\mathscr{A}_{1}:=s(x)^{-1} \mathscr{A} s(x)$ and boundary conditions defined by $B_{1}, C_{1}$ (cf. Proposition 2.2). Then $\operatorname{Det}_{\pi}\left(A_{1}\right)=$ Det ${ }_{\pi}(A)$ as the spectrum of $A$ and the operator $A_{1}$, defined by $\mathscr{A}_{1}$ and boundary conditions $\left(B_{1}, C_{1}\right)$ do coincide. By Proposition 2.2,

$$
\operatorname{det}\left(B_{1} Y_{1}(T)-C_{1}\right)=(\operatorname{det} s(T))^{-n} \operatorname{det}(B Y(T)-C) .
$$

As we have noted above, $\operatorname{det} s(T)=\exp \left\{\frac{i}{2 n} \int_{0}^{T} \operatorname{tr}\left(a_{2 n-1}(y)\right) d y\right\}$. Finally note that $\mathscr{A}_{1}$ is of the form

$$
\mathscr{A}_{1}=D^{2 n}+\sum_{k=0}^{2 n-2} a_{1 k}(x) D^{k}
$$

and then we may and will assume that for $\mathscr{A}, a_{2 n}(x) \equiv$ Id and $a_{2 n-1}(x) \equiv 0$.

Deformation 3. Applying Proposition 3.1 and Proposition 3.2 we conclude that it remains to prove that $f\left(A_{B}, C\right)=0$ for $\mathscr{A}=D^{2 n}+\lambda$ and $B, C$ given by

$$
\ell_{j}=d_{x}^{\alpha_{j}}, \quad m_{j}=d_{x}^{\beta_{j}} \quad(1 \leq j \leq n)
$$

where $\lambda$ is chosen positive and sufficiently large so that $A_{B, C}$ is $1-1$. This is verified by an explicit computation. To make writing easier we restrict ourselves to that case $r=1$. However, to obtain the explicit formulas for $g_{\alpha}$ and $g_{\beta}$ we consider $\mathscr{A}=\rho D^{2 n}+\lambda$ with $\rho>1$. Denote by $Y(x, \lambda)$ the fundamental matrix for $\rho D^{2 n}+\lambda$. For $\lambda>0$, let $\mu=\left(\frac{\lambda}{\rho}\right)^{1 / 2 n}$. Then, with $w_{k}:=\exp \left(i \frac{2 k-n-1}{2 n} \pi\right), Y(x, \lambda)$ is equal to

$$
\left(\begin{array}{ccc}
e^{\mu w_{1} x} & \cdots & e^{\mu w_{2 n} x} \\
\mu w_{1} e^{\mu w_{1} x} & \cdots & \mu w_{2 n} e^{\mu w_{2 n} x} \\
\vdots & & \vdots \\
\left(\mu w_{1}\right)^{2 n-1} e^{\mu w_{1} x} & \cdots & \left(\mu w_{2 n}\right)^{2 n-1} e^{\mu w_{2 n} x}
\end{array}\right)\left(\begin{array}{ccc}
1 & \cdots & 1 \\
\mu w_{1} & \cdots & \mu w_{2 n} \\
\vdots & & \vdots \\
\left(\mu w_{1}\right)^{n-1} & \cdots & \left(\mu w_{2 n}\right)^{2 n-1}
\end{array}\right)^{-1} .
$$

Further define $B=\left(B_{j k}\right), C=\left(C_{j k}\right)$ by

$$
\begin{aligned}
B_{j k} & = \begin{cases}1 & \text { if } 1 \leq j \leq n \text { and } k=\alpha_{j}, \\
0 & \text { otherwise }\end{cases} \\
C_{j k} & = \begin{cases}1 & \text { if } n+1 \leq j \leq 2 n \text { and } k=\beta_{j-n}, \\
0 & \text { otherwise }\end{cases}
\end{aligned}
$$

We have to show that

$$
\operatorname{Det}_{\pi}\left(\left(\rho D^{2 n}+\lambda\right)_{B, C}\right)=(-1)^{|\beta|}(2 n)^{n}\left(h_{\alpha} h_{\beta}\right)^{-1} \rho^{g_{\alpha}+g_{\beta}} \operatorname{det}(B Y(T, \lambda)-C) .
$$

For that purpose we introduce

$$
\begin{aligned}
& w(\lambda):=\log \operatorname{Det}_{\pi}\left(\left(\rho D^{2 n}+\lambda\right)_{B, C}\right), \\
& \delta(\lambda):=\log \operatorname{det}(B Y(T ; \lambda)-C) .
\end{aligned}
$$

As $n \geq 1$, we know from Proposition 3.1 that $\frac{d}{d \lambda} w(\lambda)=\frac{d}{d \lambda} \delta(\lambda)$. Therefore it suffices to consider the asymptotics of $w(\lambda)$ and $\delta(\lambda)$ as $\lambda \rightarrow+\infty$.

First recall from [Fr] (cf. also [Vo]) that $w(\lambda)$ admits an asymptotic expansion of the form $\sum_{k=-1}^{\infty} p_{k} \lambda^{-k / n}+\sum_{j=0}^{\infty} q_{j} \lambda^{-j} \log \lambda$ with the property that $p_{0}=0$. To find the asymptotics of $\delta(\lambda)$ as $\lambda \rightarrow \infty$, write $Y(T, \lambda)$ in the form

$$
Y(T ; \lambda)=L W E(L W)^{-1}
$$


where $L=\operatorname{diag}\left(1, \mu, \mu^{2}, \ldots, \mu^{2 n-1}\right), E:=\operatorname{diag}\left(e^{\mu w_{1} T}, \ldots, e^{\mu w_{2 n} T}\right)$ and

$$
W=\left(\begin{array}{ccc}
1 & \cdots & 1 \\
w_{1}^{1} & \cdots & w_{2 n}^{1} \\
\vdots & & \vdots \\
w_{1}^{2 n-1} & \cdots & w_{2 n}^{2 n-1}
\end{array}\right) .
$$

Thus $\delta(\lambda)=\log \left(\operatorname{det} W^{-1} L^{-1}\right)+\log \operatorname{det}(B L W E-C L W)$. Observe that the $(j, k)$ th coefficient of the matrix $B L W E-C L W$ is of the form $e^{\mu w_{k} T} f_{j k}(\mu)+$ $g_{j k}(\mu)$ where $f_{j k}(\mu)$ and $g_{j k}(\mu)$ are rational functions of $\mu$. We conclude that, with $\Omega=\sum_{j=1}^{n} w_{j}=\sum_{j=1}^{n} \operatorname{Re} w_{j}$,

$$
\log \operatorname{det}(B L W E-C L W)
$$

$$
=\mu \Omega T+\log \operatorname{det}\left[B L W\left(\begin{array}{cc}
\operatorname{Id}_{n} & 0 \\
0 & 0
\end{array}\right)-C L W\left(\begin{array}{cc}
0 & 0 \\
0 & \operatorname{Id}_{n}
\end{array}\right)\right]+e(\lambda)
$$

where $\lim _{\lambda \rightarrow \infty} e(\lambda)=0$. The matrix $B L W\left(\begin{array}{cc}\operatorname{Id}_{n} & 0 \\ 0 & 0\end{array}\right)-C L W\left(\begin{array}{ll}0 & 0 \\ 0 & \text { Id }_{n}\end{array}\right)$ is of the form $\left(\begin{array}{cc}F^{(1)} & 0 \\ 0 & F^{(2)}\end{array}\right)$ where $F^{(i)}$ are $n \times n$ matrices given by $(1 \leq j, k \leq n)$

$$
F_{j k}^{(1)}:=\mu^{\alpha_{j}} w_{k}^{\alpha_{j}}, \quad F_{j k}^{(2)}:=-\mu^{\beta_{j}} w_{n+k}^{\beta_{j}}=(-1)^{\beta_{j}+1} \mu^{\beta_{j}} w_{k}^{\beta_{j}}
$$

where we used that $w_{n+k}=-w_{k}$. Therefore, with $|\alpha|=\sum_{1}^{n} \alpha_{j},|\beta|=\sum_{1}^{n} \beta_{j}$

$$
\begin{gathered}
\operatorname{det}\left[B L W\left(\begin{array}{cc}
\operatorname{Id}_{n} & 0 \\
0 & 0
\end{array}\right)-C L W\left(\begin{array}{cc}
0 & 0 \\
0 & \operatorname{Id}_{n}
\end{array}\right)\right] \\
=\mu^{|\alpha|} \operatorname{det}\left(w_{k}^{\alpha_{j}}\right) \mu^{|\beta|}(-1)^{|\beta|+n} \operatorname{det}\left(w_{k}^{\beta_{j}}\right) .
\end{gathered}
$$

In view of the fact that $\left.\operatorname{det} L^{-1}\right|_{\lambda=1}=\prod_{j=0}^{2 n-1}\left(\frac{1}{\rho}\right)^{-j / 2 n}=\rho^{\frac{2 n-1}{2}}$, this implies that the 0 th order coefficient of the asymptotic expansion of $\delta(\lambda)$ for $\lambda \rightarrow \infty$ is of the form

$$
\begin{aligned}
\delta_{+\infty} & :=\left.\operatorname{det} L^{-1}\right|_{\lambda=1}+\log \left\{\operatorname{det}\left(W^{-1}\right) \operatorname{det}\left(w_{k}^{\alpha_{j}}\right)(-1)^{|\beta|+n} \operatorname{det}\left(w_{k}^{\beta_{j}}\right) \rho^{-(|\alpha|+|\beta|) / 2 n}\right\} \\
& =\log \rho^{\frac{2 n-1}{2}}-\log \rho^{(|\alpha|+|\beta|) / 2 n}+\log \left((-1)^{|\beta|+n} \operatorname{det}\left(W^{-1}\right) h_{\alpha} h_{\beta}\right)
\end{aligned}
$$

where $h_{\alpha}=\operatorname{det}\left(w_{k}^{\alpha_{j}}\right), h_{\beta} \equiv \operatorname{det}\left(w_{k}^{\beta_{j}}\right)$.

By a straightforward computation we have $\operatorname{det} W=(-1)^{n}(2 n)^{n}$ and therefore

$$
w(\lambda)=\delta(\lambda)-\delta_{+\infty}=\delta(\lambda)+\log \left\{(-1)^{|\beta|}(2 n)^{n} h_{\alpha}^{-1} h_{\beta}^{-1} \rho^{\left(\frac{|\alpha|}{2 n}-\frac{n}{2}+\frac{1}{4}+\frac{|\beta|}{2 n}-\frac{n}{2}+\frac{1}{4}\right)} .\right.
$$

The claim (3.4) then follows from the following.

Lemma 3.3. $\tilde{g}_{\alpha}=\frac{1}{2}\left(\frac{|\alpha|}{n}-n+\frac{1}{2}\right)$.

Proof. In view of Proposition 2.3 we obtain from (3.5) in the case $\alpha=\beta$

$$
2 \tilde{g}_{\alpha}=2\left(\frac{|\alpha|}{2 n}-\frac{n}{2}+\frac{1}{4}\right) \quad \text { or } \quad \tilde{g}_{\alpha}=\frac{1}{2}\left(\frac{|\alpha|}{n}-n+\frac{1}{2}\right) .
$$




\section{REFERENCES}

[BFK1] D. Burghelea, L. Friedlander, and T. Kappeler, On the determinant of elliptic differential and finite difference operators in vector bundles over $S^{1}$, Comm. Math. Phys. 138 (1991), $1-18$.

[BFK2] _ Regularized determinants for pseudodifferential operators on vector bundles over $S^{1}$, Integral Equations Operator Theory 16 (1993), 496-513.

[BFK3] _ Meyer-Vietoris type formula for determinants of elliptic differential operators, J. Funct. Anal. 107 (1992), 34-66.

[DD] T. Dreyfus and H. Dym, Product formulas for the eigenvalues of a class of boundary value problems, Duke Math. J. 45 (1978), 15-37.

[Fo1] R. Forman, Functional determinants and geometry, Invent. Math. 88 (1987), 447-493.

[Fo2] - Determinants, finite difference operators and boundary value problems, Comm. Math. Phys. 147 (1992), 485-526.

[Fr] L. Friedlander, The asymptotic of the determinant function for a class of operators, Proc. Amer. Math. Soc. 107 (1989), 169-178.

[RS] B. Ray and I. Singer, R-torsion and the Laplacian on Riemann manifolds, Adv. in Math. 7 (1971), 145-210.

[Se1] R. Seeley, The resolvent of an elliptic boundary value problem, Amer. J. Math. 91 (1969), 889-920.

[Se2] Analytic extension of the trace associated with elliptic boundary value problems, Amer. J. Math. 91 (1969), 963-983.

[Vo] A. Voros, Spectral function, special functions and Selberg zeta function, Comm. Math. Phys. 110 (1987), 439-465.

(D. Burghelea and T. Kappeler) Department of Mathematics, Ohio State University, Columbus, Оhіо 43210

(L. Friedlander) Department of Mathematics, University of Arizona, Tucson, Arizona 85721 